\title{
PROBABILISTIC BASED RELIABILITY ASSESSMENT CONCEPTS FOR COMPOSITE POWER SYSTEMS
}

\author{
Anazia E. A. ${ }^{*}$, Onyedikachi S.N. ${ }^{2}$, Enemuoh F.O. ${ }^{3}$ \\ 1,2,3 Department of Electrical Engineering, Nnamdi Azikiwe University, Nigeria \\ Ianinyemunzele@yahoo.com, ${ }^{2}$ ns.onyedikachi@gmail.com, ${ }^{3}$ eneody@yahoo.com
}

*Corresponding Author: -

Email: aninyemunzele@yahoo.com

\begin{abstract}
: -
The ability of a power system to maintain sufficient supply of electricity at all operating condition reflects its reliability. It is a measure of the systems or components adequacy and security in achieving its role at all times. This work presents system adequacy with respect to reliability following discussions on the basic probability concepts relevant for the reliability assessment of a functional composite power system (hierarchical level II). The concept has been illustrated with a simple composite system of three components; generator, transformer and transmission line. Proceeding on the assumption that components exist in one of the two-state model and not in multi-states, the steady state transition diagram was developed in order to identify system states and the transition rates between states on account of the failure or restoration of a system component. With system states and interstate transition rates identified, state and event probabilities determined, reliability indices that describes the adequacy of such composite systems can be evaluated.
\end{abstract}

Key words: - "Reliability Assessment", "Composite Systems", "State Transition", "State transition diagram", "Transition rates", "Failure rate", "Probability" 


\section{INTRODUCTION}

As has been for decades, statistical assessment of past performance and probabilistic evaluation of future performance have been recognized as indispensable for the continuous reliable system operation and the expansion planning of any electric power system. There are several quantitative techniques for reliability assessment of every aspect of the power system matching their usefulness. Enhanced computing facilities, efficient reliability database of power utilities, greater understanding of probabilistic techniques by engineers are some of the advancements made in power system planning, operation and analysis that have rendered the early deterministic techniques of reliability analysis in power systems obsolete system in order to explore probabilistic alternatives. It is instructive to note that both techniques, deterministic and probabilistic undertake reliability assessment in two aspects of system adequacy and system security. Adequacy relates to the existence of sufficient facilities within the system to satisfy the consumer load demand or system operational constraints, these include the facilities necessary to generate sufficient power and the requisite transmission and distribution facilities required to evacuate the generated power to the load point. Associated with static conditions, adequacy excludes system disturbances. Security assessment in power system relates to the ability of the system to respond to disturbances arising within the system. Most of the probabilistic techniques are in the domain of adequacy assessment. As may have been noted, every viable power systems consist of three functional zones of generation, transmission and the distribution systems which for the purpose of reliability assessment, may be combined to give three hierarchical levels with two having overlapping reach. These levels are namely hierarchical levels I, II and III [1]. Generating capacity reliability evaluation is determined at the hierarchical level I (HL I), where the total system generation is assessed for adequacy to match system total load demand while neglecting the transmission system capacity to evacuate generation to load points. The HL II consists of the generation and transmission systems and reliability assessment at this level is termed composite system or bulk transmission system evaluation. The degree of complexity of reliability assessment at the HL III level is above others as it involves the assessment of three zones at once. This complexity is facilitated by numerous number of distribution system components, which often is more than the sum of those in the HL II alone. Therefore, a less tedious alternative of complete system reliability is to undertake independent reliability assessments for the HLII and the distribution segment of the power system [2]. This Probabilistic assessment resides in the domain of adequacy, reliability analysis relates to assessing the adequacy of an existing or proposed system, including the impact of reinforcement alternatives at the transmission and generation levels [3]. These impacts are assessed by evaluating two sets of complementary indices. First is the individual bus (load point) indices which monitors the effect on individual busbars and provide input for HL III level and the other is the overall system indices which indicates the adequacy of the entire system. Appropriate reliability indices may be obtained by either the direct analytical techniques which represents the system by a mathematical model and evaluate the reliability indices from this model using mathematical solutions or by Monte Carlo simulation which estimate reliability indices by simulating the actual process and random behavior of the system by treating them as real experiments [4]. Of these methods of reliability assessment, there is no better method as each has comparative advantage over the other that can be exploited on the basis of the need of the evaluation process.

\section{A. Reliability Assessment Data Acquisition and Processing}

Although there are conflicting precedence between reliability data and reliability methodology, it is instructive to note that each must inseparably evolve [1]. Comprehensive discussions of qualitative reliability assessment would consistently lead to the discussion of the availability of appropriate data adequate for evaluation. Conceptually, data can be employed for two reasons; assessment of past performance and/or prediction of future performance. The first use;

i) identifies chronological changes in performance as well as weak areas needing reinforcement or modification

ii) establishes existing indices to validate future system reliability assessments

iii) enables previous predictions to be compared with actual operating experience

The predictive data use looks forward into future system behaviour which is achieved by transforming past experience into required future prediction. Collection of that is therefore indispensible as it forms the crux of input to relevant reliability models, technique and equations that estimate future performance, the benefits of alternative system designs, reinforcement and expansion plans, the effects of alternative operational maintenance policies and the related reliability cost/benefit of alternatives associated with the first two. Consequently, despite the enormous cost of acquiring relevant reliability data, the effort in acquisition must not only be deliberate but consistent till adequate data suitable for modeling and analysis are compiled. The data acquired must reflect the two main processes involved in component behaviour that influence system reliability assessment, namely the failure process and the restoration process [5]. Failures in power system occur due to outages such as forced outage where failed system component(s) are removed from service for repairs or maintenance or scheduled outages on account of preventive maintenance, expansion or additional reinforcement which are routinely performed to enhance system performance, efficiency and reliability. Outages significantly weaken system reliability and therefore their effect must be included in predictive reliability evaluation.

\section{B. Composite Reliability Assessment Data and Unit States}

Composite power system components as shown in the one line diagram of figure 1 all work as a single entity to ensure the reliability of supply if sufficient system component remain online. However, in power system reliability evaluation components are assumed to exist in discrete states such that the system state is a combination of the component states [6]. The failure and restoration processes vary from one component to another and for good reasons, not only are the different operational functions of each component in the composite system relevant, the exposure parameters to which each is subjected to are also captured. For instance, the data needed for the three category of composite components discussed in 
this paper involving; generating, transmission and transformers units is different for each due to their unique role and the impact of failure of any of them. For instance, a circuit breaker may be failed stuck, failed open or fully operational, a transformer or generator may be assumed to exist in the zero capacity, full capacity or one of the many intermediate capacity states while a transmission line are generally represented by the events which can cause change of state. The probabilities of residing in states are the statistics used for generating units while the rate or frequencies for encountering states are the statistics used for transmission facilities [7]. Therefore, the state of a composite system is a combination of the constituent mutually exclusive states, and due to the techniques for quantitative assessment involved, the reliability modeling and evaluation of such a system should proceed with the discussion of basic probability and reliability concepts for the constituent systems. Therefore, reliability analysis for composite system or HL II reliability assessment is primarily concerned with the evaluation of the states of the components and their corresponding probabilities [8].

\section{Stochastic and Deterministic Data}

The data associated with outage, failure and restoration processes has stochastic form as they relate to the random nature of events which can be measured in ways like;

- counting the number of relevant events that occur in a defined period

- measuring the time elapsed between entering and exiting a state and

- counting the number of failures to operate that occur following a given number of command to operate.

However, to completely evaluate statistical indices from stochastic data, it essential to determine relevant exposure parameters that has deterministic form which includes; number of operating components of each designated type, length of lines and cables, exposure time, discrete exposure relating to the frequency of failure. The relevance of these exposure parameters is that they relate to the denominator of the equations from which statistical indices are evaluated.

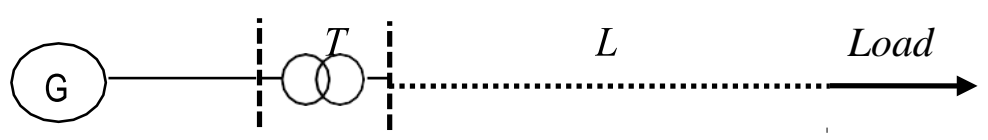

Fig 1: One line diagram of a power system

\section{PROBABILITY CONCEPTS FOR COMPOSITE RELIABILITY ASSESSMENT}

Consider the diagram in figure 1 which is essentially a power system supplying power to a load through a transmission line $(\mathrm{L})$ from a generator $(\mathrm{G})$ whose output voltage is stepped up by the transformer $(\mathrm{T})$.

This proceeds on the assumption that each composite component exist in a two-state mode of either a Down (D) or an Up (U) state and not in multi- intermediary states. Reliability analysis is concerned with the evaluation of these states and determining their probabilities. Below are some concepts relevant for reliability assessment especially for composite systems.

\section{A. Events}

An event is essentially a set of system states and defines an occurrence of a state. For instance, the event that two system components of figure 1 are UP is the event defined by the set

$$
A_{I I}^{U}=\left\{\left(G^{D} T^{U} L^{U}\right),\left(G^{U} T^{D} L^{U}\right),\left(G^{U} T^{U} L^{D}\right)\right\}
$$

Similarly, the event that all three components are DOWN is the set

$$
A_{I I I}^{U}=\left\{\left(G^{D} T^{D} L^{D}\right)\right\}
$$

Events can be mutually exclusive if they can occur together or non-mutually exclusive if the contrary holds. Similarly, events can be independent or dependent.

\section{B. State Space}

The set of all possible events of the system states is state space. In other words, all possible combinations of component states form the state of the system. Consequently, the composite system state space is,

$$
S=\left\{\left(G^{v} T^{v} L^{v}\right),\left(G^{D} T^{v} L^{v}\right),\left(G^{v} T^{D} L^{v}\right),\left(G^{v} T^{v} L^{D}\right),\left(G^{D} T^{D} L^{v}\right),\left(G^{D} T^{v} L^{D}\right),\left(G^{v} T^{D} L^{D}\right),\left(G^{D} T^{D} L^{D}\right)\right\}(3)
$$

As seen above, three composite system components produce elements in the state space. Generally, for any system of components and assuming a two-state model, there would be elements in the state space set.

\section{Probability}

This is a number existing between 0 and 1 corresponding to states and is indicative of their relative likelihood of occurrence. The probability of a system state space must always sum to unity. The combinational property of events include that they obey addition, multiplication and the complementary rules.

i) Addition rule of Probabilities: Two or more events are mutually exclusive if they cannot occur together. For instance, the events that a component is UP or DOWN are two complementary events and therefore mutually exclusive. Furthermore, if events $X_{a}, X_{b}$ and $X_{c}$ that are not mutually exclusive, then 


$$
P\left(X_{a} \cup X_{b} \cup X_{c}\right)=P\left(X_{a}\right)+P\left(X_{b}\right)+P\left(X_{c}\right)-P\left(X_{a} \cap X_{b} \cap X_{c}\right)
$$

where

$P\left(X_{a} \cup X_{b} \cup X_{c}\right)=$ The probability of $X_{a}$ or $X_{b}$ or $X_{a}$ and $X_{b}$ and

If events $X_{a}, X_{b}$ and mutually exclusive, meaning they cannot happen together, that is $P\left(X_{a} \cap X_{b} \cap X_{c}\right)=0$, Then equation (4) reduces to

$$
\text { - PCar } \left.x_{b} \cap x_{0}\right)
$$

i) Multiplication rule of Probabilities: If the probability of the occurrence of event $X_{a}$ is affected by the occurrence of $X_{c}$, then events $X_{a}$ and 4 are dependent events. The conditional probability of event given that event $X_{a}$ had occurred is denoted by $P\left(X_{c} \mid X_{a}\right)$ and then

$$
P\left(X_{a} \cap X_{c}\right)=P\left(X_{a}\right) \times P\left(X_{c} \mid X_{a}\right)
$$

Equation (6) is used to evaluate the conditional probability from reference [9] as

$$
P\left(X_{c} \mid X_{a}\right)=\frac{P\left(X_{a} \cap X_{c}\right)}{P\left(X_{a}\right)}
$$

However, when events $X_{a}$ and $X_{c}$ are independent that is the occurrence of $X_{a}$ does not affect the occurrence of $X_{c}$, then equations (6) becomes;

$$
P\left(X_{a} \cap X_{c}\right)=P\left(X_{a}\right) \cdot P\left(X_{c}\right)
$$

iii) Complementary rule of Probabilities: For event $X_{a}$ its complementary of event is denote by either $\overline{X_{a}}$ or $\overline{X_{a}{ }^{\prime}}$. The complement $\overline{X_{a}}$ is the set of states that are not members of $X_{a}$. For instance, the events that a component like a generator is UP or DOWN are $G^{U}$ and $G^{D}$ respectively and are two complementary events and therefore mutually exclusive. Mathematically, the relationship between complementary events holds as

$$
P\left(\overline{X_{a}}\right)=1-P\left(X_{a}\right)
$$

Then the relationship for the complementary events of the generator is

$$
P\left(G^{D}\right)=P\left(\overline{G^{U}}\right)=1-P\left(G^{U}\right)
$$

\section{Exponential Distribution}

While there are other probability distribution theories and approaches to describe random variables yet the one widely used in reliability analysis is the exponential distribution.

From [2], the exponential distribution of random variables $x$ is given by

$$
f(x)=\lambda e^{-\lambda x}
$$

with $\lambda$ as a positive constant, the probability distribution becomes

$$
\begin{aligned}
& f(x)=\int_{0}^{x} \lambda \mathrm{e}^{-\lambda y} d y \\
& f(x)=1-e^{-\lambda x}
\end{aligned}
$$

The mean value of $X$ which is a discrete random variable

$$
\begin{gathered}
E(X)=\int_{0}^{\infty}\left(x h \mathrm{e}^{-\lambda x}\right) d x \\
E(X)=1 / \lambda
\end{gathered}
$$

Where $\lambda$ is called the Failure rate (down time) and is the reciprocal of the mean up time if only $X$ denotes the up time of a component. Exponential distribution has been assumed adequate for describing the up time of electronic and electromechanical components over their useful life and this stems from an interesting property of the exponential distribution. Given $X$, the up time of any component, then at any time $t$, the probability distribution of the residual life time $(X-t)$, is same as that of the original $X$. In other words, the failure rate $\lambda$ is constant implying that the component does not age [10]. 


\section{E Reliability Indices}

Several indices are used in composite power system reliability but the basic indices from which others can be derived are namely probability of failure, frequency of failure and duration of failure.

i) Probability of failure: This is the steady state probability that a system or component is out of service due to failure. This is termed the long run fraction of time spent by the system or component in the failed state.

ii) Frequency of system failure: This is defined as the mean number of system failure per unit time.

iii) Mean duration of system failure: This defined as the long time average duration of a single failure.

\section{State Space Approach for Reliability Calculations}

Based on the concept of Markov processes, the state space is about the most versatile method for reliability assessment. Its direct application is limited to systems with few components. However, it can be greatly extended by applying it to subsystems and combining the results for the entire systems [11]. The state space reliability assessment proceeds on the following assumptions;

a) Each component exist in up (U) or in the down (D) states and are complementary events.

b) Components are independent.

The steps for state space probability evaluation are illustrated with a simple composite system of fig. 1 and from [2] are Identify system states

Determine system interstate transition rate Evaluate state probabilities

i) Identify the System States: This is the first step involving the identification of all possible system states resulting from the failure of the various subsystems or components. It proceeds on the assumption that components exist either in up (U) or in the down (D) states without intermediaries and that components are independent. For instance, figure 2 contains 8 possible states. Generally, a system $n$ of components results in $2^{n}$ possible states generating an equation for each state.

ii) Determine interstate transition rate: Once the first is complete identifying the interstate transition rates follows. The transition rate from state $a$ to state $b$ is the mean rate of the system passing from state $a$ to $b$ Consider figure 2, the system transits from state $\mathbf{V}$ to state $\mathbf{I}$ with the failure of the generator component and transits from state III to state IV by the repair of the transmission line L. If the state residence times are exponentially distributed, then the transition from state $\mathrm{V}$ to state $\mathrm{I}$ is the failure rate of the generator component which is $\lambda_{G}$.

iii) Evaluate State Probabilities: Following the assumption of component independence in the step above, the state probabilities can be formed by the multiplication rule.

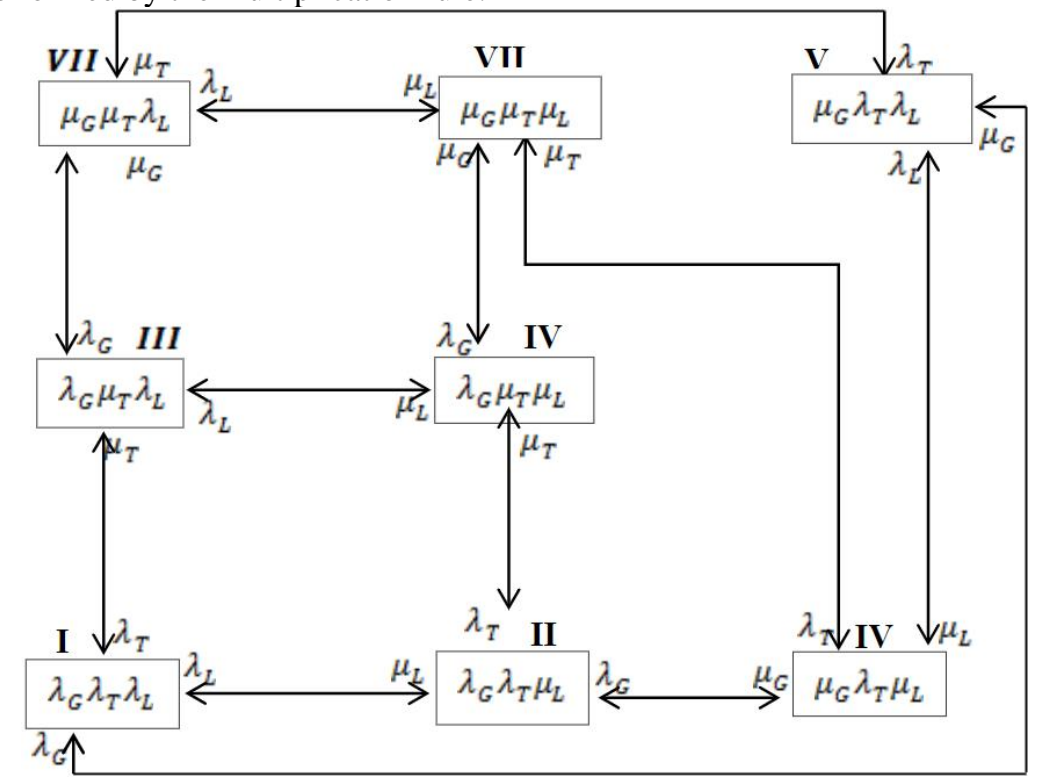

Fig 2: State Transition diagram of a typical composite system with three components

$$
P(G \cap T \cap L)=P(G) P(T) P(L)
$$

However, with statistical dependence, a set of simultaneous equations must be solved to evaluate the state probabilities. For instance, the probability expression for each of the odd numbered state in the transition table of fig. 2 are

$$
\begin{aligned}
& P_{I}=P\left(G^{D}\right) \times P\left(T^{D}\right) \times P\left(L^{D}\right) \\
& P_{I I}=P\left(G^{D}\right) \times P\left(T^{U}\right) \times P\left(L^{D}\right) \\
& P_{V}=P\left(G^{U}\right) \times P\left(T^{D}\right) \times P\left(L^{D}\right)
\end{aligned}
$$




$$
P_{\text {VII }}=P\left(G^{U}\right) \cdot P\left(T^{U}\right) \cdot P\left(L^{D}\right)
$$

If the probability of being UP or DOWN for any composite component $X$ is $P\left(X^{U}\right)$ and $P\left(X^{D}\right)$ respectively, their values can be evaluated using (15) and (16) given $\mu_{x}$ and $\lambda_{x}$ as the repair/restoration rate and failure rate of component

$$
\begin{gathered}
P\left(X^{U}\right)=\frac{\mu_{x}}{\lambda_{x}+\mu_{x}} \\
P\left(X^{D}\right)=\frac{\lambda_{x}}{\lambda_{x}+\mu_{x}}
\end{gathered}
$$

For the composite system shown in fig 1, if the assumed probabilities values of being UP for the generator and transmission line are 0.9214 and 0.8732 respectively and the probability of being DOWN for the transformer is 0.0655 , then the complement for each derives from

$$
\begin{aligned}
& P\left(G^{D}\right)=P\left(\overline{G^{U}}\right)=1-P\left(G^{U}\right)=0.0786 \\
& P\left(L^{D}\right)=P\left(\overline{L^{U}}\right)=1-P\left(L^{U}\right)=0.1268 \\
& P\left(T^{U}\right)=P\left(\overline{T^{D}}\right)=1-P\left(T^{D}\right)=0.9345
\end{aligned}
$$

Given

$$
P\left(G^{U}\right)=0.9214, P\left(L^{U}\right)=0.8732 \text { and } P\left(T^{D}\right)=0.0655
$$

Therefore the probability values the odd numbered states is:

$$
\begin{aligned}
& P_{I}=0.0786 \times 0.0655 \times 0.8732=0.004495 \\
& P_{I I I}=0.0786 \times 0.9345 \times 0.1268=0.009314 \\
& P_{V}=0.9214 \times 0.0655 \times 0.1268=0.007653 \\
& P_{V I I}=0.9214 \times 0.9345 \times 0.1268=0.109181
\end{aligned}
$$

It is instructive to note that evaluating the state probabilities of dependent components would require the solution of a set of simultaneous equations. For a system of remponents each with two states of UP or DOWN only (assuming no

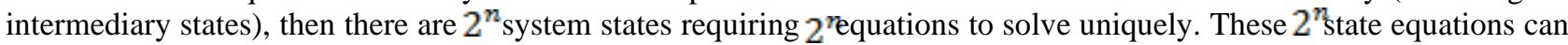
be written conveniently using the concept of frequency balance [10] represented as;

$$
\sum_{\forall a \neq b} E_{a b}=\sum_{\forall a=b} E_{b a}
$$

Where

The frequency of transiting from state $a$ to $b$ is

$$
E_{a b}=P_{a} \gamma_{a b}
$$

$P_{a}=$ Probability of a system state $a$

$\gamma_{a b}={ }_{\text {Transition rate from state }}^{a}$ to $b$ given

The implication of equation (17) is that, for steady state, the frequency of exiting from a state ${ }^{a}$ equals the frequency of entering the state ${ }^{a}$.

\section{A. Transition Rate Matrix}

Returning to the state transition diagram of fig.2,

For state I

The Frequency entering state

$$
\mathbf{I}=P_{V} \lambda_{G}+P_{I I I} \lambda_{T}+P_{I I} \lambda_{L}
$$

The Frequency of existing state

$$
\mathbf{I}=P_{I}\left(\mu_{G}+\mu_{T}+\mu_{L}\right)
$$

Now for the eight states, the corresponding equations (19a) - (19h) are 


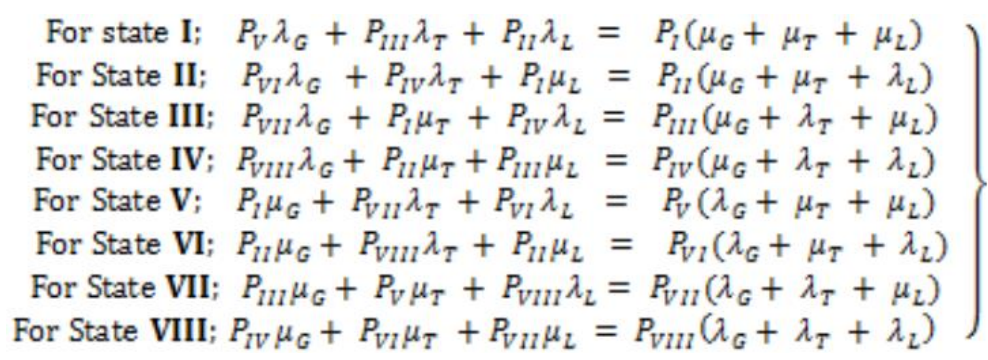

But recall that In other words

In other words

$$
\sum P_{a}=1
$$

$$
P_{I}+P_{I I}+P_{I I I}+P_{I V}+P_{V}+P_{V I}+P_{V I I}+P_{V I I I}=1
$$

It is noteworthy that while $2^{n}$ equations are needed to compute state probabilities, the $2^{n}$ equations obtained from equation (17) are not linearly independent and there is only $2^{n}-1$ along equation (20) would be used.

Hence equations (19a) - (19h) and equation (21) can be solved to obtain the state probabilities which proceeds as For the LHS of equation (19a) - (19h) in matrix form is

$$
\left[\begin{array}{cccccccc}
0 & \lambda_{L} & \lambda_{T} & 0 & \lambda_{G} & 0 & 0 & 0 \\
\mu_{L} & 0 & 0 & \lambda_{T} & 0 & \lambda_{G} & 0 & 0 \\
\mu_{T} & 0 & 0 & \lambda_{L} & 0 & 0 & \lambda_{G} & 0 \\
0 & \mu_{T} & \mu_{L} & 0 & 0 & 0 & 0 & \lambda_{G} \\
\mu_{G} & 0 & 0 & 0 & 0 & \lambda_{L} & \lambda_{T} & 0 \\
0 & \mu_{G} & 0 & 0 & \mu_{L} & 0 & 0 & \lambda_{T} \\
0 & 0 & \mu_{G} & 0 & \mu_{T} & 0 & 0 & \lambda_{L} \\
0 & 0 & 0 & \mu_{G} & 0 & \mu_{T} & \mu_{L} & 0
\end{array}\right]\left[\begin{array}{c}
P_{I} \\
P_{I I} \\
P_{I I I} \\
P_{I V} \\
P_{V I} \\
P_{V I I} \\
P_{V I I I}
\end{array}\right]
$$

While the RHS of equation (19a) - (19h) in matrix form and numbered (23) is

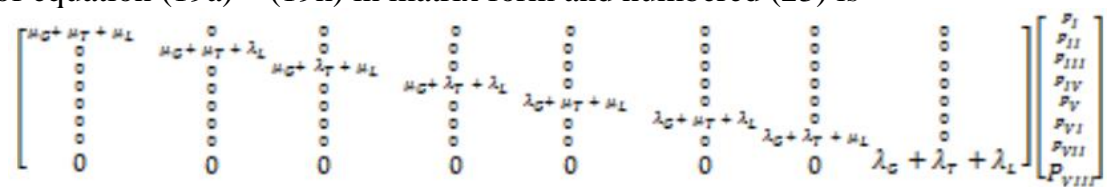

Subtracting equation (23) from equation (23) to give equation (24) as

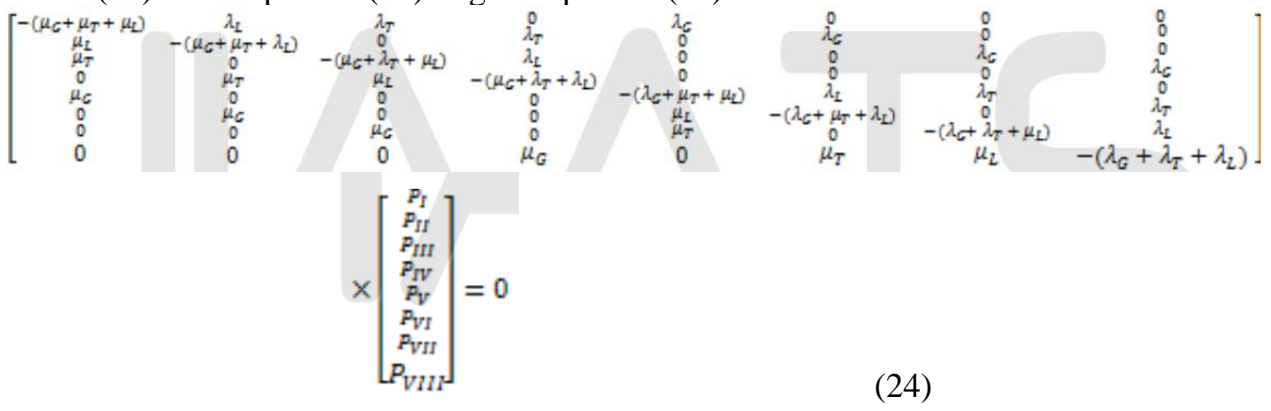

And in compact matrix form, equation becomes

$$
[R][P]=0
$$

Where

$$
[P]=\left[\begin{array}{llllllll}
P_{I} & P_{I I} & P_{I I I} & P_{I V} & P_{V} & P_{V I} & P_{V I I} & P_{V I I I}
\end{array}\right] \text { and }
$$

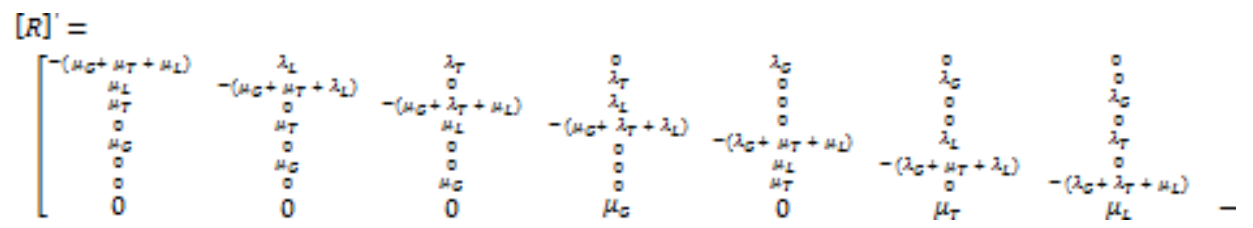

Since $[P] \neq 0$ then $[R]^{\circ}$ the matrix of the transpose of $[R]$ is zero; $[R]^{\circ}=0$ 


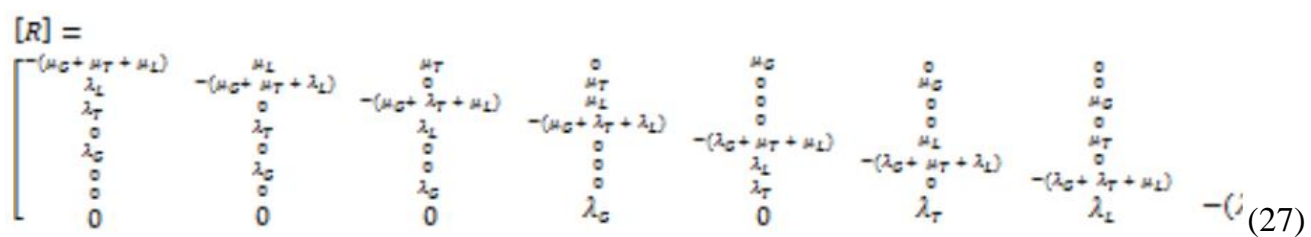

Equation (27) is the Transition rate matrix for the composite system of three components. In the same vein, equations (19a) - (19h) and (21) can be written in the form

$$
W P=S
$$

Where

$W=$ Matrix obtained from $[R]^{\prime}$ by replacing the elements of an arbitrarily selected row $k$ by 1

$P=$ Column vector whose $a^{\text {th }}$ term $P_{a}$ is the steady state probability of the system being in state $a$

given as $[P]$ above

$S=$ Column Vector with $k^{\text {th }}$ element equal to 1 and other elements set to 0

$W=$

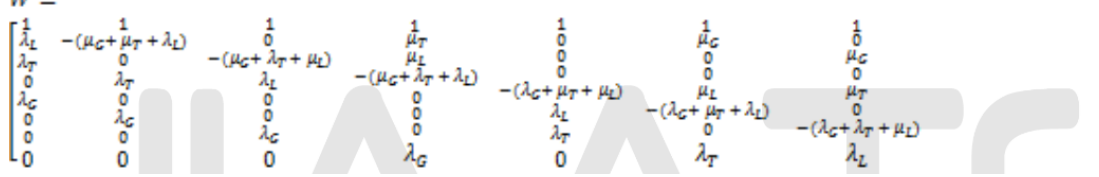

In our case, if $k=1$, then the matrix $\mathrm{W}$ stated above,

$$
S=\left[\begin{array}{llllllll}
1 & 0 & 0 & 0 & 0 & 0 & 0 & 0
\end{array}\right]
$$

\section{B. Reliability Measure}

While it is possible to maintain system component for continued supply, system reliability is significantly affected by the failure of a single component in the topology of figure 1. The failure of the transformer means that the output of an online generator cannot get to the transmission line. Similarly, the failure of a transmission line means that the transformed voltage of the generator output cannot be evaluated to the load centre. Below is a summary of the possible outage/failure events for the composite topology given in one-line diagram of fig. 1

\section{Table I}

\begin{tabular}{|c|c|c|c|}
\hline $\begin{array}{l}\text { Event } \\
\text { Identificatio } \\
\text { n }\end{array}$ & $\begin{array}{l}\text { Event } \\
\text { description }\end{array}$ & $\begin{array}{ll}\text { States } & \\
& \text { involve } \\
\text { d } & \text { in } \\
\text { defined Event }\end{array}$ & $\begin{array}{l}\text { Reliability } \\
\text { Remark }\end{array}$ \\
\hline $\begin{array}{l}\text { Event } \\
\text { A: }\end{array}$ & $\begin{array}{l}\text { One component } \\
\text { failed }\end{array}$ & $\{I V, V I, V I I\}$ & - \\
\hline Event B: & $\begin{array}{l}\text { Two } \\
\text { Components } \\
\text { failed }\end{array}$ & $\{I I, I I I, V\}$ & - \\
\hline Event $C$ : & $\begin{array}{l}\text { All components } \\
\text { failed }\end{array}$ & $\{I\}$ & \begin{tabular}{|rr} 
Worst & \\
& sys \\
& tem reliability
\end{tabular} \\
\hline Event D: & $\begin{array}{l}\text { No Component } \\
\text { failed }\end{array}$ & $t\{V I I I\}$ & \begin{tabular}{|rr} 
Best & \\
& sys \\
tem reliability
\end{tabular} \\
\hline
\end{tabular}

Three Component Composite System Outage Event Probability

Therefore the probability of any event $M$ equals

$$
P_{M}=\sum_{a \in M} P_{a}
$$

The frequency of event $M$ event equals

$$
f_{M}=\sum_{a \in(s-M)} P_{a} \sum_{b \in M} \gamma_{a b}
$$


The mean duration of event $\mathrm{M}$ equals

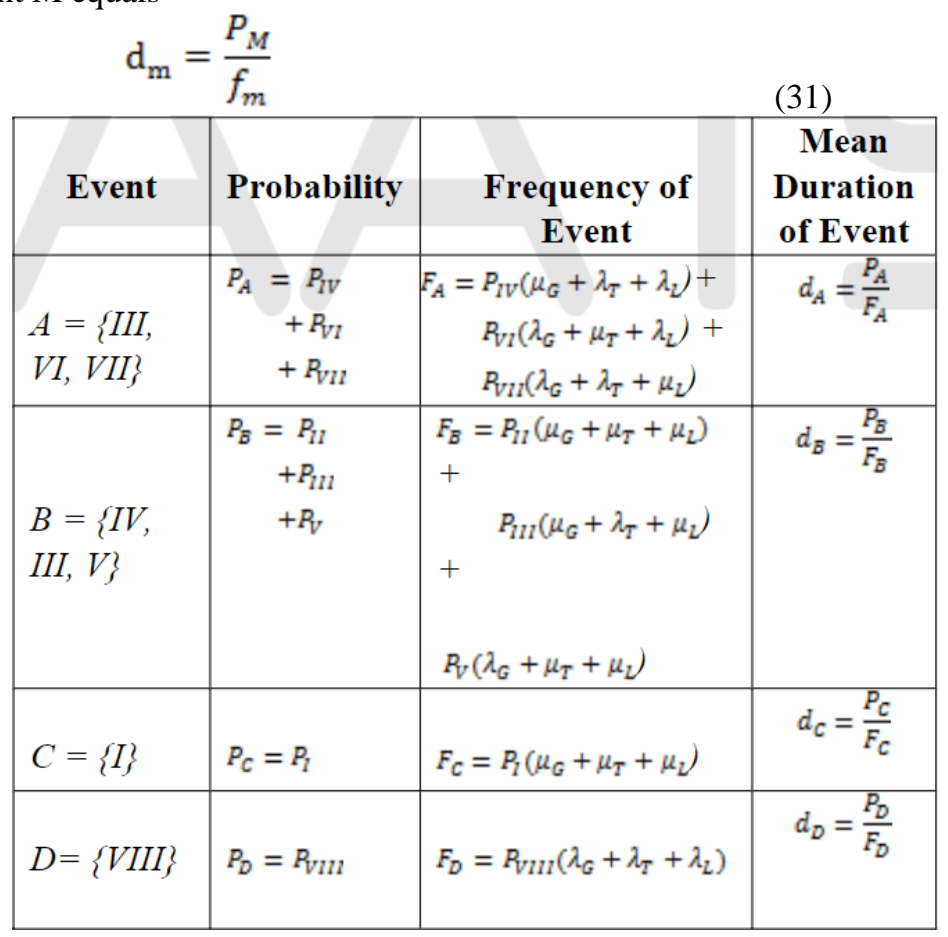

\section{CONCLUSION}

The reliability index for composite power systems involving generation and transmission components have been illustrated using probabilistic approach achieved by defining the probable events and their probability values, the frequency of these events with respect to the failure rates from the transition table and then their corresponding mean duration of each event as shown in the last table.

\section{REFERENCES}

Allan R.N., Billinton R. \& Lee S.H. (1984). "Bibliography on the Application of Probability Methods" in Power System Reliability evaluation. IEEE Transaction PAS-103, 275-282.

[1]. Allan, R. B. \& Billinton, R.. "Reliability Concepts of Composite Power System" in Reliability Assessment of Composite Generation and Transmission Systems, IEEE Tutorial Course, 90EH0311-1-PWR, pp 5-8. 1989

[2]. Schilling, M. Th., El-Kady, M. A., Billinton, R. \& Leite da Silva, A. M. "Bibliography on Composite Reliability (1964 -1988)". IEEE Winter Power Meeting, New York, 1989, paper 89 WM 190-0-PWRS

[3]. Allan, R. N.. "Concepts of Data for Assessing the Reliability of Composite Systems". in Reliability Assessment of Composite Generation and Transmission Systems, IEEE Tutorial Course, 90EH0311-1-PWR, pp 14-20, 1989

[4]. Singh C. "Basic Probability and Reliability Concepts". Reliability Assessment of Composite Generation and Transmission Systems, IEEE Tutorial Course, 90EH0311-1-PWR, 1989 pp 9- 13.

[5]. Billinton Roy. "Bibliography on the Applications of probability Methods in Power Sysytem Reliability Evaluation". IEEE Trans., PAS-91, 1972 pp 649-660

[6. IEEE Committee Report, "Common Mode Forced Outages of Overhead Transmission lines". IEEE Trans. PAS-95, 1976, pp 859-863

[7]. CIRGE Working Group 38.03, " Power System Reliability Analysis: Application Guide". CIGRE, 1988

[8]. Billinton R. \& Allan R. N. "Reliability Evaluation of Power Systems". Plenum Press, New York, 1984

[9]. Stroud K.A. \& Dexter J. B. "Engineering Mathematics". 6 ${ }^{\text {th }}$ Ed. Palgrave Macmillan New York, pp 1139-1213. 2007

[10].Billinton R. \& Singh C. "System Reliability Modelling and Evaluation". Hutchinson Educational, London. 1977

[11].Fong, C. C. "Bulk System Reliability Measurement and Ontario Hydro Experience". in Reliability Assessment of Composite Generation and Transmission Systems, IEEE Tutorial Course, 90EH0311-1-PWR, 1989 pp 21-28 